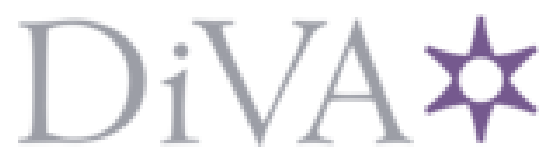

http://www.diva-portal.org

\title{
Postprint
}

This is the accepted version of a paper presented at SSP 2016, June 26-29, Palma de Mallorca, Spain.

Citation for the original published paper:

Halvorsen, K., Olsson, F. (2016)

Pose estimation of cyclic movement using inertial sensor data.

In: Proc. 19th Statistical Signal Processing Workshop IEEE Signal Processing Society https://doi.org/10.1109/SSP.2016.7551830

N.B. When citing this work, cite the original published paper.

Permanent link to this version:

http://urn.kb.se/resolve?urn=urn:nbn:se:uu:diva-307229 


\section{POSE ESTIMATION OF CYCLIC MOVEMENT USING INERTIAL SENSOR DATA}

\author{
K. Halvorsen*. \\ Department of Information Technology \\ Uppsala University \\ Uppsala, Sweden \\ Email: kjartan.halvorsen@it.uu.se \\ and \\ Department of Mechatronics \\ Instituto Tecnologico y de Estudias Superiores \\ de Monterrey, Atizapan, Mexico
}

\author{
F. Olsson \\ Department of Information Technology \\ Uppsala University \\ Uppsala, Sweden \\ Email: fredrik.olsson@it.uu.se
}

\begin{abstract}
We propose a method for estimating the rotation and displacement of a rigid body from inertial sensor data based on the assumption that the movement is cyclic in nature, meaning that the body returns to the same position and orientation at regular time intervals. The method builds on a parameterization of the movement by sums of sinusoids, and the amplitude and phase of the sinusoids are estimated from the data using measurement models with Gaussian noise. The maximum likelihood estimate is then equivalent to a weighted nonlinear least squares estimate. The performance of the method is demonstrated on simulated data and on experimental data.
\end{abstract}

Index Terms - Motion capture, MEMS, IMU, Accelerometer, Gyro, Maximum likelihood

\section{INTRODUCTION}

Many human movements are cyclic in nature, in particular locomotion. Finding repeated patterns in the kinematics of cyclic motion has been the focus of several studies [1,2]. In this work the main goal is not the detection of cyclic patterns per se, but rather to use the assumption of cyclic motion as a way of regularizing the problem of estimating the pose from inertial sensor data, where drift in the estimates is a common problem.

We model the cyclic movement as a sum of sinusoids, i.e. a truncated Fourier series. This type of model has previously been used in studies of cardiac motion [3] and in robotics [4]. The truncated Fourier-series model of a periodic signal is a natural choice, and the expressive power of the model can easily be tuned by choosing the number of terms included in the series.

\section{ESTIMATING CYCLIC ROTATION}

\subsection{Parameterization of rotation}

The orientation of the rigid body can be described by a unit quaternion

$$
q=\left[\begin{array}{ll}
r & v
\end{array}\right]^{\mathrm{T}}=\left[\begin{array}{llll}
r & v_{1} & v_{2} & v_{3}
\end{array}\right]^{\mathrm{T}}
$$

*This work was supported by the Swedish Research Council (project number 2015-05054). The authors acknowledge Dr Dennis Sturm for providing experimental data. where $r$ is the scalar part and $v$ is the vector part. When the quaternion operates on a vector $a$ in the body frame it yields the vector in the static frame:

$$
a^{s}=q a^{b} q^{*}
$$

where $q^{*}$ is the conjugate of the unit quaternion $q$ (and hence also its inverse). Superscript ${ }^{s}$ denotes a vector in a static reference frame (static frame), and superscript ${ }^{b}$ a vector in a frame fixed to the moving body (body frame). Quaternion multiplication between the factors is implied. Vectors and points in 3D are represented with a zero scalar part. See [5] for further details on quaternion algebra.

As a minimal parameterization of rotation, we will make use of the stereographic projection of unit quaternions. This projection maps any point on the northern hemisphere $(r>0)$ of the unit sphere in $4 \mathrm{D}$ onto the unit disk in $3 \mathrm{D}$ intersecting the $4 \mathrm{D}$ unit sphere at the equator $(r=0)$. The rotation can thus described by three parameters

$$
\psi=\left[\begin{array}{lll}
\rho & \phi & \chi
\end{array}\right]^{\mathrm{T}}
$$

with the origin corresponding to the identity quaternion (no rotation). There are many alternative parameterizations of rotations in three parameters, and the rationale for choosing stereographic projection is that it gives rational functions for the mapping between the parameters and the unit quaternion

$$
q=\frac{1}{\lambda^{2}+1}\left[\begin{array}{llll}
1-\lambda^{2} & 2 \rho & 2 \phi & 2 \chi
\end{array}\right]^{\mathrm{T}}
$$

where $\lambda^{2}=\rho^{2}+\phi^{2}+\chi^{2}$.

\subsection{Model of cyclic rotation}

We define a frame fixed to the body and with orientation that coincides with the static frame at the beginning of each cycle. In the $\psi$ space this means that all trajectories start and end at the origin. The cyclic motion is modeled as a finite number, $m$, of harmonic sine waves with base frequency $w$, assumed to be known. The motion is thus parameterized by the amplitude and phase of these sinusoids. Considering the model of the $\rho$ component of $\psi$, a periodic signal with mean-value zero is

$$
\begin{aligned}
\bar{\rho}(t) & =\sum_{k=1}^{m}\left(\alpha_{\rho_{k}} \sin (k w t)+\beta_{\rho_{k}} \cos (k w t)\right) \\
& =\left[\begin{array}{ll}
s^{\mathrm{T}}(t) & c^{\mathrm{T}}(t)
\end{array}\right]\left[\begin{array}{l}
\alpha_{\rho} \\
\beta_{\rho}
\end{array}\right]
\end{aligned}
$$


where

$$
\begin{aligned}
c^{\mathrm{T}}(t) & =\left[\begin{array}{llll}
\cos w t & \cos 2 w & \cdots & \cos m w t
\end{array}\right], \\
s^{\mathrm{T}}(t) & =\left[\begin{array}{llll}
\sin w t & \sin 2 w & \cdots & \sin m w t
\end{array}\right], \\
\alpha_{\rho} & =\left[\begin{array}{llll}
\alpha_{\rho_{1}} & \alpha_{\rho_{2}} & \cdots & \alpha_{\rho_{m}}
\end{array}\right]^{\mathrm{T}}, \\
\beta_{\rho} & =\left[\begin{array}{llll}
\beta_{\rho_{1}} & \beta_{\rho_{2}} & \cdots & \beta_{\rho_{m}}
\end{array}\right]^{\mathrm{T}} .
\end{aligned}
$$

However, in order to obtain a signal that starts in the origin we subtract the sum of the amplitudes of the cosines

$$
\rho(t)=\bar{\rho}(t)-\sum_{k=1}^{m} \beta_{\rho_{k}}
$$

The parameterizations of $\phi(t)$ and $\chi(t)$ are similar.

\subsection{Signal model}

Most inertial measurement units (IMUs) contains tri-axial gyros and magnetometers. For the gyro signal, we assume a discrete-time model including bias and zero-mean noise

$$
y_{g}\left(t_{k}\right)=\omega^{b}\left(t_{k}\right)+b_{g}+v_{g}\left(t_{k}\right)
$$

where

$$
\omega^{b}(t)=2 q^{*}(t) \dot{q}(t) .
$$

is the angular velocity of the body (in body coordinates), $b_{g}$ is the gyro bias and $v_{g}\left(t_{k}\right)$ is a white, Gaussian noise sequence with covariance $\Sigma_{g}$, assumed to be constant and known.

To utilize the magnetometer for orientation estimation, we store the measurement of the magnetic field at the start of the very first cycle in the vector $y_{m_{0}}$. The magnetometer measurements are assumed to be unbiased, since such a bias is impossible to detect without other measurements of absolute orientation. We consider a zeromean measurement noise sequence, which gives the following model for the magnetometer signal

$$
y_{m}\left(t_{k}\right)=q^{*}\left(t_{k} ; \theta\right) y_{m_{0}} q\left(t_{k} ; \theta\right)+v_{m}\left(t_{k}\right),
$$

where $y_{m_{0}}$ is the magnetometer reading of the first sample. The white, Gaussian noise term $v_{m}\left(t_{k}\right)$ has covariance $\Sigma_{m}$, assumed to be constant and known.

\subsection{Estimation}

Assuming independent noise sequences in the gyro and magnetometer signals, the log-likelihood function of the unknown parameters

$$
\theta=\left[\begin{array}{lllllll}
b_{g}^{\mathrm{T}} & \alpha_{\rho}^{\mathrm{T}} & \beta_{\rho}^{\mathrm{T}} & \alpha_{\phi}^{\mathrm{T}} & \beta_{\phi}^{\mathrm{T}} & \alpha_{\chi}^{\mathrm{T}} & \beta_{\chi}^{\mathrm{T}}
\end{array}\right]^{\mathrm{T}}
$$

given the sensor data

$$
Y=\left\{y_{g}\left(t_{0}\right), \ldots, y_{g}\left(t_{N-1}\right), y_{m}\left(t_{0}\right), \ldots, y_{m}\left(t_{N-1}\right)\right\}
$$

becomes

$$
\begin{aligned}
\ell(\theta ; Y)= & -\frac{1}{2} \sum_{k=0}^{N-1}\left\|y_{g}\left(t_{k}\right)-\omega^{b}\left(t_{k}\right)-b_{g}\right\|_{\Sigma_{g}^{-1}}^{2} \\
& -\frac{N}{2} \log 2 \pi-\frac{N}{2} \log \operatorname{det} \Sigma_{g} \\
& -\frac{1}{2} \sum_{k=0}^{N-1}\left\|y_{m}\left(t_{k}\right)-q^{*}\left(t_{k} ; \theta\right) y_{m_{0}} q\left(t_{k} ; \theta\right)\right\|_{\Sigma_{m}^{-1}}^{2} \\
& -\frac{N}{2} \log 2 \pi-\frac{N}{2} \log \operatorname{det} \Sigma_{m}
\end{aligned}
$$

We make the further assumption that the noise covariances are known. A reasonable estimate can be found by computing the covariance matrices from a sequence of sensor data with the sensors at rest. Under this assumption, maximizing the log-likelihood function leads to the nonlinear least squares problem

$$
\begin{aligned}
\underset{\theta}{\arg \min } & \frac{1}{2} \sum_{k=0}^{k=N-1} \epsilon_{k}(\theta)^{\mathrm{T}} W_{g} \epsilon_{k}(\theta) \\
& +\frac{1}{2} \sum_{k=0}^{k=N-1} e_{k}(\theta)^{\mathrm{T}} W_{m} e_{k}(\theta),
\end{aligned}
$$

where,

$$
\begin{aligned}
\epsilon_{k}(\theta) & =y_{g}\left(t_{k}\right)-\omega^{b}\left(t_{k} ; \theta\right)-b_{g} . \\
e_{k}(\theta) & =y_{m}\left(t_{k}\right)-q\left(t_{k} ; \theta\right) y_{m_{0}} q^{*}\left(t_{k} ; \theta\right), \\
W_{g} & =\Sigma_{g}^{-1} \\
W_{m} & =\Sigma_{m}^{-1} .
\end{aligned}
$$

The minimization problem can be solved using the Gauss-Newton algorithm, for which the Jacobian matrix

$$
J_{k}=\left[\begin{array}{ll}
\frac{\partial \epsilon_{k}^{\mathrm{T}}}{\partial \theta} & \frac{\partial e_{k}^{\mathrm{T}}}{\partial \theta}
\end{array}\right]^{\mathrm{T}}
$$

is needed. The Jacobian can be approximated by discrete differences or calculated in explicit form. We chose to derive the Jacobian explicitly (details omitted), since it leads to faster convergence.

\section{ESTIMATING CYCLIC POSE}

\subsection{Model of cyclic displacement}

Similarly to the model (6) of the rotation, we propose a model of cyclic displacement of the inertial sensor node as a truncated Fourier series with base frequency $w$. The number of terms in the series can differ for the model of orientation and the model of displacement. We assume the origin of the body frame and the static frame to coincide at the start of each cycle. The model for the displacement in the $x$-direction becomes

$$
x(t)=\left[\begin{array}{ll}
s^{\mathrm{T}}(t) & c^{\mathrm{T}}(t)
\end{array}\right]\left[\begin{array}{l}
\alpha_{x} \\
\beta_{x}
\end{array}\right]-\sum_{k=1}^{n} \beta_{x_{k}}
$$

and similarly for $y(t)$ and $z(t)$. Derivatives of the displacements are straightforward to calculate.

\subsection{Signal model}

The models for the gyro signal and the magnetometer signal are given in (7) and (9), respectively. We assume that the accelerometer measurements are bias-free, but corrupted by a zero-mean measurement noise sequence. This gives the model

$$
y_{a}\left(t_{k}\right)=q^{*}\left(t_{k} ; \theta\right)\left(a\left(t_{k} ; \theta\right)+g\right) q\left(t_{k} ; \theta\right)+v_{a}\left(t_{k}\right),
$$

where

$$
a\left(t_{k} ; \theta\right)=\left[\begin{array}{l}
\ddot{x}\left(t_{k} ; \theta\right) \\
\ddot{y}\left(t_{k} ; \theta\right) \\
\ddot{z}\left(t_{k} ; \theta\right)
\end{array}\right],
$$

is the acceleration of the sensor in the spatial frame, $g$ is the gravity vector and $v_{m}\left(t_{k}\right)$ the sensor noise with covariance $\Sigma_{a}$. 


\subsection{Estimation}

The parameter vector contains the bias of the gyro, gravity and the parameters for the rotation and displacement:

$$
\theta=\left[b_{g}, \alpha_{\rho}, \beta_{\rho}, \alpha_{\phi}, \beta_{\phi}, \alpha_{\chi}, \beta_{\chi}, g, \alpha_{x}, \beta_{x}, \alpha_{y}, \beta_{y}, \alpha_{z}, \beta_{z}\right] .
$$

Assuming independent noise sequences in the three sensors and known noise covariances, the maximum likelihood estimate is given by the solution to the nonlinear least squares problem

$$
\begin{aligned}
\underset{\theta}{\arg \min } & \frac{1}{2} \sum_{k=0}^{k=N-1} \epsilon_{k}(\theta)^{\mathrm{T}} W_{g} \epsilon_{k}(\theta) \\
& +\frac{1}{2} \sum_{k=0}^{k=N-1} e_{k}(\theta)^{\mathrm{T}} W_{m} e_{k}(\theta) \\
& +\frac{1}{2} \sum_{k=0}^{k=N-1} d_{k}(\theta)^{\mathrm{T}} W_{a} d_{k}(\theta)
\end{aligned}
$$

where

$$
\begin{aligned}
d_{k}(\theta) & =y_{a}\left(t_{k}\right)-q^{*}\left(t_{k} ; \theta\right)\left(a\left(t_{k} ; \theta\right)+g\right) q\left(t_{k} ; \theta\right), \\
W_{a} & =\Sigma_{a}^{-1} .
\end{aligned}
$$

\section{EXAMPLES}

The method was implemented in Python (version 2.7.10) on SageMathCloud [6].

\subsection{Simulated data}

A cyclic movement of a rigid body was created using a sum of eight sinusoids both for orientation and displacement. The amplitudes and phases of the sinusoids were chosen randomly, sampling from a uniform distribution between 0 and 0.5 for the amplitudes and uniform 0 to $2 \pi$ for the phase. Data sets consisting of 200 samples completing two cycles were simulated according to the sensor models (7), (9) and (14). Uncorrelated Gaussian noise was added to the data. For the gyro the standard deviation (std) was between 0.01 and $0.13 \mathrm{rad} / \mathrm{s}$, and for the magnetometer std between 0.01 and 1 . This latter noise is unitless, since it is added to the normalized magnetometer reading, then normalized again. For the gyro data a random (but constant over the sequence) bias term was also added of magnitude $0.1 \mathrm{rad} / \mathrm{s}$. For each condition (noise variance) 30 different data sequences were generated with different noise realizations.

Figure 1 shows the Root Mean Square error in orientation when the noise in the gyro is increased. The orientation estimation deterioriates as the noise in the gyro data increases. Note that in the criterion function (11) the residuals in the gyro- and magnetometer measurements are weighed with the inverse of the noise covariance. Hence, as the gyro noise increases, the measurements carry less weight in the minimization. Consequently, the algorithm relies more and more on the magnetometer data, which are invariant for rotations around an axis aligned with the (local) magnetic north. This is a likely reason for the deterioration in figure 1. Increasing noise in the magnetometer data had much less influence on the orientation estimation (data not shown).

To illustrate pose estimation, we simulated a case with noise of similar variance as in the experimental data described in the next section. The true and estimated displacements are illustrated in figure 2. It should be noted that the displacement estimates are rather sensitive to sensor noise, and also to model error if the number of terms in the model is less than that of the true data (results not shown).

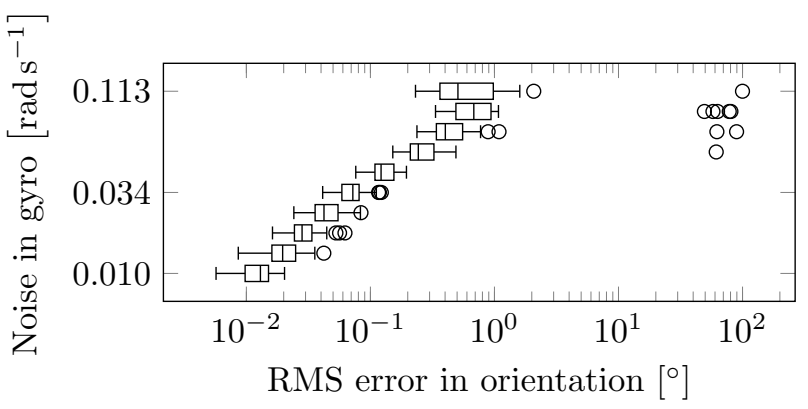

Fig. 1. Root mean square error in orientation for different levels of noise in the gyro. Simulated data with 200 samples containing two complete movement cycles.

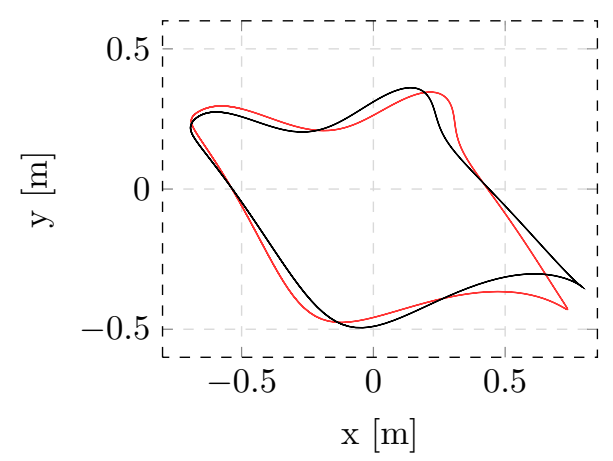

Fig. 2. Estimated displacement (in 3D projected onto the $x y$-plane) using simulated data with realistic noise variance, and no model error. The red line shows the true displacement, and the black shows the estimate. 


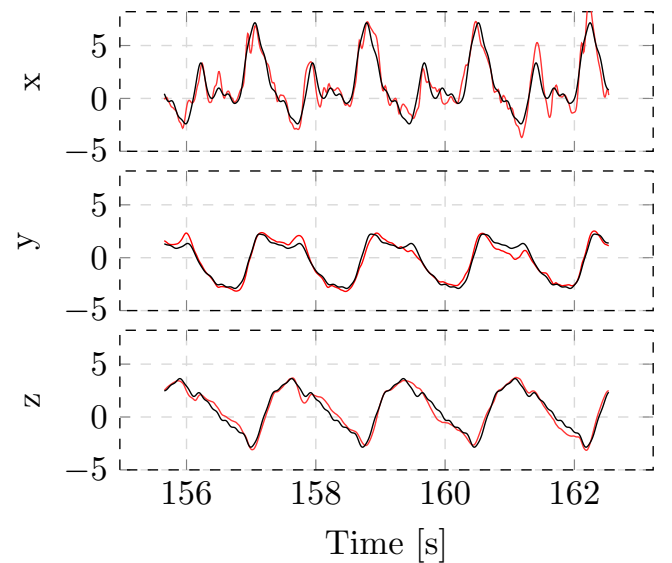

Fig. 3. Measured gyro data (red) and estimated cyclic data (black) using a model with 12 harmonics. The unit is $\mathrm{rad} / \mathrm{s}$.

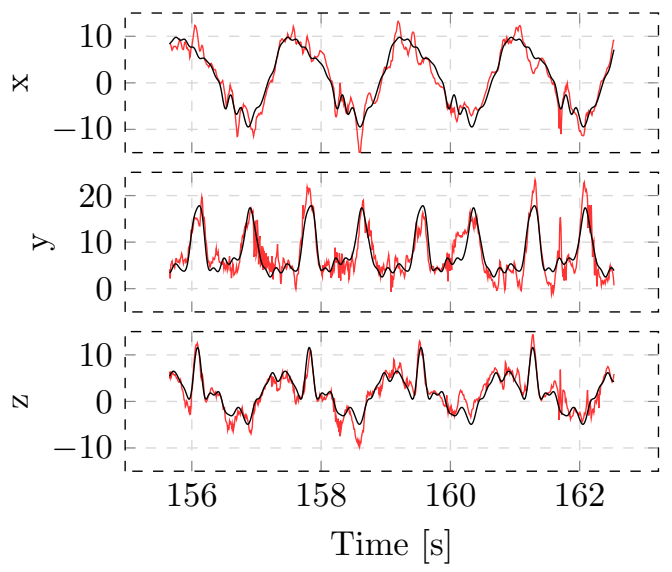

Fig. 4. Measured accelerometer data (red) and estimated cyclic data (black) using a model with 12 harmonics. The unit is $\mathrm{m} / \mathrm{s}^{2}$.

\subsection{Experimental data}

The experimental data is comprised of gyro- and accelerometer data from a custom sensor node [7] attached to a kayak paddle. The sensor node also includes a force transducer which was used to detect the start of each cycle (the force peaks at insertion into the water). The node was attached to a kayak paddle just inside of the left hand. The sensor's $\mathrm{x}$-axis is aligned with the paddle shaft and the $\mathrm{y}$-axis is normal to the plane of the paddle blade.

A recreational athlete paddled at a steady stroke rate at medium intensity in measurement sequences of about one minute each. Data from four complete cycles was extracted from the middle of a measurement sequence, containing 705 samples at a sampling period of $9.77 \mathrm{~ms}$

Figures 3, 4 and 5 show measured data and model output using a model with 12 harmonics for both the cyclic rotation and for the displacement. The pattern and magnitude of the displacement as seen in figure 5 is typical of kayak paddling [8]. No validation data was available for this data sequence, however.

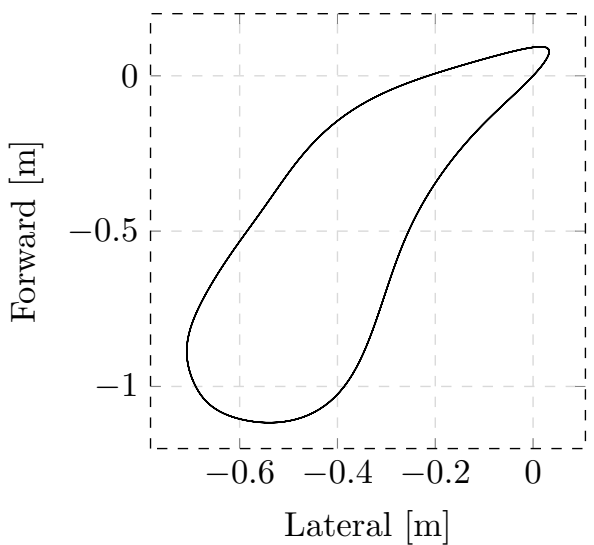

Fig. 5. Estimated displacement of sensor (left hand) in the horizontal plane as seen from above.

\section{CONCLUSIONS AND FUTURE WORK}

The simulation results show that the method is effective in recovering cyclic movement from IMU data corrupted by realistic noise and bias. The displacement estimate is more sensitive to sensor noise and modelling errors than the orientation estimate. This property should be investigated further. The results from the real data of a kayak paddle movement indicate that the method can generate cyclic movement which reproduces the salient features of the sensor data (figures 3 and 4). Important applications of the method include ambulatory analysis of human locomotion and technique in endurance sports. Although involving a nonlinear least squares minimization over 100+ variables, the method converges in less than the duration of the data sequence. Hence, it could be used for analysis and feedback in real-time to the individual.

A validation against gold-standard motion capture utilizing an industrial robot arm is in progress. The assumption of a fixed, known base frequency is a strong one, hence future work includes extending the method to also estimate a slowly changing base frequency.

\section{REFERENCES}

[1] D. Ormoneit, M. J. Black, T. Hastie, and H. Kjellström, "Representing cyclic human motion using functional analysis," Image and Vision Computing, vol. 23, no. 14, pp. 1264-1276, 2005.

[2] V. P. Stokes, H. Lanshammar, and A. Thorstensson, "Dominant pattern extraction from 3-d kinematic data," Biomedical Engineering, IEEE Transactions on, vol. 46, no. 1, pp. 100-106, 1999.

[3] J. C. McEachen, A. Nehorai, and J. S. Duncan, "Multiframe temporal estimation of cardiac nonrigid motion," Image Processing, IEEE Transactions on, vol. 9, no. 4, pp. 651-665, 2000.

[4] K. McIsaac, J. P. Ostrowski et al., "Motion planning for anguilliform locomotion," Robotics and Automation, IEEE Transactions on, vol. 19, no. 4, pp. 637-652, 2003.

[5] J. B. Kuipers et al., Quaternions and rotation sequences. Princeton university press Princeton, 1999, vol. 66.

[6] T. S. Developers, Sage Mathematics Software (Version 6.10), 2015, http: /cloud. sagemath. com. 
[7] D. Sturm, "Wireless multi-sensor feedback systems for sports performance monitoring," $\mathrm{Ph} . \mathrm{D}$. dissertation, $\mathrm{PhD}$ thesis, $\mathrm{PhD}$ Thesis, KTH Royal Institute of Technology, 2012.

[8] S. J. Kendal and R. H. Sanders, "The technique of elite flatwater kayak paddlers using the wing paddle," $J A B$, vol. 8, no. 3, 2010. 